\title{
Protée
}

\section{Rhétorique des figures visuelles et argumentation par images dans le discours scientifique}

\section{Maria Giulia Dondero}

Volume 38, numéro 1, printemps 2010

Le Groupe $\mu$ entre rhétorique et sémiotique

URI : https://id.erudit.org/iderudit/039701ar

DOI : https://doi.org/10.7202/039701ar

Aller au sommaire du numéro

\section{Éditeur(s)}

Département des arts et lettres - Université du Québec à Chicoutimi

ISSN

0300-3523 (imprimé)

1708-2307 (numérique)

Découvrir la revue

Citer cet article

Dondero, M. G. (2010). Rhétorique des figures visuelles et argumentation par images dans le discours scientifique. Protée, 38(1), 41-53.

https://doi.org/10.7202/039701ar
Résumé de l'article

Notre propos consiste à comparer une rhétorique des figures visuelles, comme nous l'a proposée le Groupe $\mu$ dans son Traité du signe visuel (1992), avec une rhétorique de l'argumentation par images - qui, bien que beaucoup moins connue, a produit un certain nombre d'études au sein de la sémiotique du discours d'inspiration greimassienne. Cette dernière approche vise à dépasser l'étude du trope en tant que figure locale allotopique et à rendre compte des enjeux argumentatifs produits par la disposition et l'enchaînement discursifs d'images et textes verbaux à l'échelle du discours. Si les effets rhétoriques on souvent été étudiés sur des ouvrages artistiques, notre objectif est de nous interroger sur le syncrétisme du discours scientifique. Notre étude se termine en fait par une analyse de l'iconographie des trous noirs dans un ouvrage de vulgarisation scientifique d'astrophysique. 


\title{
RHÉTORIQUE DES FIGURES VISUELLES ET ARGUMENTATION PAR IMAGES DANS LE DISCOURS SCIENTIFIQUE
}

\author{
MARIA GIULIA DONDERO \\ Il se peut qu'un énoncé littéralement faux soit vrai métaphoriquement.
}

(Goodman, 1992: 28)

\section{INTRODUCTION}

Ce travail s'articule en deux volets: le premier sera consacré à parcourir les avancées de la rhétorique visuelle dans les deux traditions sémiotiques européennes: celle du Groupe $\mu$ et celle de l'École de Paris. Il s'agira aussi de voir comment les deux traditions ont (ou non) donné une place à l'analyse du discours scientifique. Le second volet sera consacré à l'étude de la rhétorique visuelle dans un ouvrage de vulgarisation scientifique en astrophysique.

Nous nous proposons donc non seulement d'esquisser quelques pistes de réflexion du côté du visuel concernant les différences et les similitudes entre les deux néorhétoriques, mais aussi d'apporter une contribution à la question débattue de la puissance argumentative et cognitive de l'image. D'ailleurs, une fois admise l'idée que l'image est articulée comme le langage verbal en plan d'expression et plan du contenu grâce à l'instance médiatrice de l'énonciation, et que cette instance module les effets de personne, espace et temps de l'image elle-même, on peut aussi accepter l'idée que l'image est capable de condenser et déployer un raisonnement, ainsi que de préfigurer des hypothèses scientifiques. À cette fin, nous prendrons comme objet d'étude la mise en scène d'objets scientifiques très particuliers, tels les trous noirs en physique théorique, par le travail d'un astrophysicien qui, en premier, en a étudié l'iconographie: Jean-Pierre Luminet de l'Observatoire de Paris-Meudon. Il s'agit d'un cas très intéressant à notre sens, puisque les trous noirs sont des objets dont l'existence n'est que postulée.

\section{DEUX APPROCHES SÉMIOTIQUES DE LA RHÉTORIQUE VISUELLE}

On peut, nous semble-t-il, envisager la rhétorique visuelle de deux manières: la première conçoit l'image comme susceptible d'être traversée par des traits allotopiques et comme productrice de figures tropiques (c'est plutôt la voie parcourue par le Groupe $\mu$ ); la deuxième saisit l'image - ou bien la série d'images et de textes verbaux - à l'intérieur d'un flux argumentatif (c'est plutôt la voie d'une sémiotique 
de l'énonciation et du discours). Tout en n'étant pas directement liée au modèle de la rhétorique de l'argumentation de Perelman, cette seconde conception de la rhétorique a en commun avec la rhétorique argumentative la taille pertinente de l'analyse: le discours et non la figure isolée. Si la première a travaillé finement sur les sous-unités iconiques et plastiques des images et sur les opérations grammaticales qui en constituent l'architecture rhétorique - centrée sur le rapport norme/écart -, la seconde chercherait plutôt à analyser le rapport translocal entre isotopies et allotopies à l'intérieur d'un tissu textuel à la fois verbal et visuel. Cela ne veut pas dire que la rhétorique de figures ne soit pas intéressée au rapport verbo-visuel, tout au contraire, mais il faut dire que ce rapport a été saisi de manière différente par les deux rhétoriques: dans le premier cas, l'attention a été portée sur la composition sémantique des sous-unités verbo-visuelles composant une figure et sur leurs agencements; dans le second cas, l'attention a été portée sur les stratégies énonciatives des langages syncrétiques, notamment sur les opérations de débrayage et d'embrayage, voire d'assertion et d'assomption ${ }^{1}$. La conception de la rhétorique du discours est liée à la polyénonciation et à la relation entre passages discursifs mis en tension sur le plan des visées énonciatives et des hiérarchisations des voix, englobantes et englobées. Si la rhétorique visuelle des figures a été beaucoup fréquentée par les sémioticiens grâce à l'ouvrage majeur du Groupe $\mu$ (1992) qui a fait école, et aux travaux de Göran Sonesson (2007, 2010), la rhétorique visuelle de l'argumentation n'a pas eu de véritable institutionnalisation et ses acteurs se sont rarement déclarés rhétoriciens ${ }^{2}$. En fait, comme on vient de le dire, il s'agit d'une rhétorique qui ne descend pas des travaux de Perelman et de ses successeurs, mais d'une autre tradition savante: celle de la théorie sémiotique de l'énonciation d'A.J. Greimas (la rhétorique de l'elocutio, on le sait, est la science des mises en œuvre du langage) ${ }^{3}$. La première rhétorique s'est consacrée à étudier les jeux de langage verbo-visuels dans les œuvres d'art et dans les images publicitaires, qui sont en effet les textes qui se prêtent le mieux à la mise en scène des écarts des normes et à la créativité dans le système langagier visuel. La seconde rhétorique pourrait se définir, quant à elle, comme une rhétorique de l'argumentation centrée sur l'analyse des stratégies énonciatives en grande partie consacrée, elle aussi, au discours publicitaire (Floch, Marrone, Basso Fossali) et au discours artistique (Floch, Fabbri, Beyaert-Geslin, Le Guern), mais également à la communication politique (BeyaertGeslin, Landowski), aux discours juridique (Jackson) et scientifique - qui sont les domaines où l'argumentation vise spécialement à négocier la distance conflictuelle entre les partenaires de l'action discursive ${ }^{4}$.

On pourrait affirmer que la rhétorique de la science en Europe a été inaugurée par Paolo Fabbri et Bruno Latour dans un article portant sur ce sujet (1977) et avec les travaux de Françoise Bastide $(1985,2001)$ portant sur les stratégies énonciatives des discours visuel et verbal dans les articles scientifiques et dans les revues de vulgarisation. Cette rhétorique de la science aurait l'ambition d'étudier l'argumentation visuelle et verbale comme un "tout de signification" de deux manières: non seulement par l'étude d'une commensurabilité possible des valeurs au niveau profond du parcours génératif du contenu, mais aussi, au niveau de la surface discursive, par l'étude de la comparabilité des formes pronominales verbales et visuelles (la soi-disant intersubjectivité en discours) et la commensurabilité aspectuelle des parcours visuels et verbaux. En ce qui concerne la comparabilité des formes pronominales verbales et visuelles, il y a une longue tradition qui remonte aux théoriciens de l'image qui ont été les pères de la sémiotique visuelle de l'école greimassienne (Lhote, 1958; Paris, 1969; Marin, 1993; Damisch, 2000; Florenskij, 1995; Schapiro, 2000, etc.), mais aussi à un certain nombre de sémioticiens contemporains (Shaïri et Fontanille, 2001; Fabbri, 2004 et 2009, etc.) qui ont expliqué la relation entre le rapport intersubjectif «je-tu ", dans le discours verbal et la représentation du visage de face dans le discours visuel, et le rapport impersonnel engendré par l'utilisation du «il» et du portrait de profil au sein d'une relation semi-symbolique. De 
surcroît, Anne Beyaert-Geslin, dans plusieurs articles et dans l'ouvrage récent L'Image préoccupée (2009), a avancé des propositions en expliquant la différence visuelle entre la forme du «il» (représentation du visage de face) et la forme visuelle du "on" (représentation de la tête) dans la photographie artistique et de reportage politique.

À propos de cette comparabilité des formes pronominales dans l'image, et dans le champ plus restreint des images scientifiques, Bastide a longuement écrit sur les différentes manières qu'ont les scientifiques de cadrer et recadrer les photographies et de mettre en valeur les traces des données pour légitimer leur argumentation. La focalisation sur certaines données, qui les dispose au centre de la topologie de l'image et de manière la plus éclairée possible, nette - et nettoyée des artefacts -, construit un effet d'impersonnalité et d'objectivation qui correspond aux procédures énonciatives objectivantes de la troisième personne (on) utilisées dans le texte verbal qui accompagne ces images. L'effet d'indifférencié visuel, voire les images entourées par des vagues, des contours flous et incertains, est toujours utilisé rhétoriquement pour démasquer le désordre d'une vision subjective (le je des autres) - et peu adaptée au discours scientifique - attribuée aux collègues rivaux dont les théories sont remises en question dans l'article. En outre, en ce qui concerne l'aspectualité (inchoativité, durativité, terminativité, ponctualité), la régulation du zoom dans les séries et dans les enchaînements d'images peut construire différents types d'aspectualité perceptive (la régularité durative, la découverte ponctuelle, etc.), selon les traits et les configurations visuelles qu'on souhaite mettre en valeur. Bastide remarque que ces formes de temporalisation visuelle correspondent aux aspectualités des verbes utilisés dans la description des procédures d'investigation.

Étant donné que, comme l'affirme Bastide, c'est le caractère bien délimité du voir qui nous permet d'acquérir le savoir, il s'agira, dans une rhétorique du discours scientifique, de décrire les montages et les démontages des formes et des traits, les actions d'ajouter et d'écarter, d'inclure et d'exclure, bref de sélectionner: trouver les bonnes frontières entre traits visuels pour faire apparaittre les bonnes configurations (voir Allamel-Raffin, 2010). Par exemple, pour Bastide, il y a deux stratégies pour faire apparaître le différencié (visuel et cognitif):

1) repérer à l'intérieur de l'«indifférencié» de nouvelles limites et des frontières pour faire apparaître un objet différencié initialement trop restreint, en l'enrichissant progressivement tout au long d'un parcours de plus en plus figuratif, peutêtre à travers des stratégies d'aspectualisation;

2) restreindre un champ trop élargi et renvoyer au champ de l'indifférencié tout ce qu'on n'arrive pas à expliquer, voire faire de l'indifférencié un bruit de fond: peut-être en éliminant les acteurs des transformations ou en montrant qu'ils ne jouent aucun rôle dans le parcours figuratif qui est en train de se construire.

On s'aperçoit très vite que Bastide décrit la valeur du cadrage dans la production d'un objet cognitif à travers des procédures qui nous rappellent les opérations rhétoriques fondamentales, ces dernières étant communes aux deux rhétoriques mentionnées plus haut (l'addition, la suppression, la substitution, la dissociation, la conjonction, etc.).

D'ailleurs, on pourrait affirmer que, d'une certaine façon, non seulement Bastide a décrit la rhétorique textuelle (au sens greimassien) des articles scientifiques, elle a aussi esquissé la description d'une rhétorique $d u$ faire des scientifiques qui, encore aujourd'hui, reste à développer ${ }^{5}$. Elle a traité notamment la question de la focalisation du détail, en remarquant que, en peinture comme dans le phénomène naturel, il n'y a pas d'unités distinctes préalablement données - comme cela arrive avec les mots dans le discours verbal -; il faut plutôt fixer les limites pour isoler le détail dans le continuum constitué par «tout ce qui se passe» lors d'une expérience. Dans le cadre d'une expérience, Bastide prend ainsi en compte non seulement une coupure spatiale, mais aussi une coupure temporelle. La fréquence établie pour des observations de transformations est un exemple de coupure arbitraire d'une unité dans le continuum du déroulement temporel: le phénomène cognitif est le résultat de coupures et de focalisations 
spatiales et temporelles qui doivent ensuite être verbalement et visuellement bricolées. On reviendra plus loin sur une possible rhétorique du faire et du geste.

\subsection{La notion de conflit dans les deux rhétoriques}

Pour revenir aux points de divergence et de commensurabilité des deux manières de pratiquer la rhétorique visuelle ${ }^{6}$, on se demandera comment il serait possible aujourd'hui d'étudier le discours scientifique en tirant parti des deux traditions de pensée. On souhaite montrer, comme l'a déjà argumenté Jean-Marie Klinkenberg (2008) en prenant des exemples de comparabilité provenant du discours notamment verbal, qu'il n'est pas nécessaire d'opposer de manière définitive la rhétorique des figures (qui met en jeu un conflit sémantique) et la rhétorique de l'argumentation (dont le conflit repose sur les variations de l'assomption énonciative), car les deux partagent les mêmes objectifs fondamentaux (la renégociation d'une opposition par des techniques médiatrices, le dynamisme de l'encyclopédie, etc.)?

Comme il est couramment admis, le discours scientifique est peut-être l'un des plus conflictuels et donc rhétoriques entre tous. Comme le rappelle Bastide, chaque affirmation est la contre-affirmation d'une théorie soutenue par d'autres scientifiques ${ }^{8}$, et chaque image doit être justifiée par l'ensemble de ses paramètres d'énonciation - qui doivent pouvoir être répétables.

Si les deux rhétoriques sont centrées sur l'identification du conflit (et maintes fois de sa résolution), elles le sont de deux points de vue différents. La rhétorique des figures identifie dans certaines manifestations visuelles un écart par rapport à une norme codifiée par un système catégoriel, mais aussi par une encyclopédie, plus ou moins locale, plus ou moins liée au style de l'auteur, à un certain corpus, à l'intertexte, etc., ou bien simplement à ce que Sonesson appelle "le monde de la vie», tandis que la rhétorique énonciative vise surtout à identifier et expliquer des conflits entre stratégies énonciatives, narratives, thymiques et passionnelles appartenant à des formes de vie différentes. D'ailleurs, ce n'est pas forcément l'écart en soi qui intéresse la sémiotique d'inspiration greimassienne, parce qu'elle vise à analyser non pas des unités auxquelles sont appliquées les opérations rhétoriques fondamentales, mais plutôt les différentes formes d'assomption des oppositions catégorielles sur lesquelles les discours se fondent. Ces formes d'assomptions concernent la prise de position de l'énonciateur et du modèle implicite de l'énonciataire et constituent les valences déterminant les valeurs des arguments et des méréologies discursives. D'une certaine manière, on pourrait dire que la rhétorique des figures joue sur un système d'oppositions fixées avant le déploiement discursif, qui, par conséquent, en déterminent le sens rhétorique; la rhétorique de l'énonciation, en revanche, fait en sorte que ce sont ce qu'on appelle les valences des valeurs localement inscrites qui codéterminent le système des oppositions, les valences des valeurs étant précisément les différentes manières d'assumer les oppositions valoriales par les différentes voix énonciatives. D'ailleurs, ce sont les modes d'existence qui intègrent et modulent la question de l'assomption énonciative, qui permettent de rendre compte non seulement des multiples couches discursives - qui ne se résolvent pas en unités -, mais aussi de leurs modulations réciproques - qui ne se résolvent pas en simples substitutions. Comme l'a montré Fontanille, l'effet tropique se joue sur la relation entre modes d'existence sémiotiques, ce qui permet de rendre compte précisément de l'épaisseur du discours (la "profondeur figurale $d u$ discours"):

La co-présence des contenus concurrents en même temps que leur différence de statut discursif dans la figure seraient assurées par le fait qu'en un même point de la chaîne chacun des deux contenus serait doté d'un mode d'existence propre, de sorte qu'il n'y aurait pas littéralement "alternative» ou "substitution», mais co-présence dans l'épaisseur du discours, dans la profondeur des modes d'existence. (1996: 48)

Cette conception valorise non pas forcément l'opposition entre la présence et l'absence de traits visuels manifestés, mais les degrés intensifs de la cohésion des figures actoriales qui stratifient les simulacres discursifs:

Attribuer le "contenu littéral» ou le "degré conçu» au mode virtuel, c'est faire trop de cas du système, et pas assez $d u$ 
discours, et ce d'autant plus qu'en matière d'image, le système - en tant que virtualité schématique et indépendante des énonciations particulières - se dérobe sans cesse. Dans le collage de Max Ernst, commenté par le Groupe $\mu$ dans son Traité du signe visuel, et qui place une tête d'oiseau sur un corps humain, la tête humaine (contenu littéral) n'est ni absente ni virtuelle, dans la mesure où elle est elle-même impliquée par l'isotopie figurative dominante du discours visuel. Étant lui-même une création du discours en acte, ce contenu littéral doit appartenir à un mode d'existence qui relève de la praxis énonciative: ce contenu «tête humaine» est convoqué à partir des virtualités du système de représentation figuratif, puis il est actualisé par l'isotopie du discours, et enfin potentialisé pour entrer en tension avec le contenu "tête d'oiseau», qui, lui, est (apparemment) réalisé. [...] Les modes actualisé et potentialisé, notamment dans l'exemple ci-dessus, rendent tout particulièrement compte, respectivement, de l'indirection et de la redirection intentionnelles du degré conçu: indirection, parce que l'actualisation de "tête humaine» n'est qu'une feinte: elle n'arrivera pas à la réalisation; redirection, parce qu'elle dévie vers la potentialisation. (Ibid.: 48-49)

Cette conception permet de penser la catégorie absence/présence comme «relative, perceptive et modale; elle organise le champ de présence "imaginaire" d'une instance de discours, coextensif de l'univers sémiotique d'une énonciation" (ibid.: 50; nous soulignons).

1.2 Vers une rhétorique du geste et des tons discursifs Afin de déployer les questions concernant le champ de l'énonciation et des degrés de présence perceptive de la sémiotique post-greimassienne, tout en essayant de développer les enseignements du Traité $d u$ signe visuel du Groupe $\mu$, il serait envisageable d'étudier les images à partir des outils offerts par l'analyse aspectuelle et du tempo: qu'il s'agisse d'écart ou de «torsion" (Ricœur), il faudrait préciser à quel moment de l'exploration visuelle cet écart advient (avec une cadence inchoative ou bien terminative, etc.) et sur quel mode (parvenir, survenir, etc.). D'ailleurs, l'image fixe telle que le tableau, d'un point de vue génératif, n'a rien à voir avec l'appréhension simultanée $^{9}$ : si le Groupe $\mu$ a souvent été tenté d'affirmer, selon la tradition esthétique ancienne, que l'image appartient aux arts de l'espace et de la simultanéité, il a aussi offert un certain nombre de suggestions pour démentir cette affirmation, en théorisant précisément la relation entre le support de l'écriture picturale, la (ou les) matière(s) concernée(s) dans la relation entre support et apport, et surtout la manière de traiter cette relation entre support et apport. Cette théorisation, et notamment la notion de texture, a permis aux membres du groupe de rendre pertinent, pour l'analyse, l'inscription des traces de la production de l'image, dont l'image ellemême témoigne (énonciation énoncée), à partir de la répétition fictive, lors de la contemplation, des gestes productifs (énonciation en acte) qui sont au cœur des effets texturaux ${ }^{10}$. Tout cela a permis d'envisager l'image comme un support perceptif et sémantique de deux parcours de sens: celui du producteur, et celui de l'observateur qui refait, même fictionnellement, le parcours du producteur en déployant l'espace perceptivement élastique de l'image fixe (voir Dondero, 2009b).

Une autre tentative d'aller au-delà d'une sémiotique de l'image, cette dernière entendue comme objectivation textuelle, selon les mots de la sémiotique greimassienne pour rendre compte d'une rhétorique du geste productif, est faite dans le Traité du signe visuel à travers la notion de stylisation, à savoir la modification homogénéisante des propriétés globales des entités pour y «introduire des éléments provenant du producteur d'image, et qui ne sont rien de moins qu'un modèle d'univers" (1992: 307; nous soulignons). Cette stylisation est ce que la sémiotique greimassienne pourrait appeler la conduite énonciative globale d'un discours constituant une forme de vie (qui coïncide avec ce que le Groupe $\mu$ appelle ici modèle d'univers). Cette forme de vie se caractérise comme une convergence valoriale de traits expressifs et sémantiques repérables à différents niveaux de pertinence de l'analyse sémiotique: texte, objet, scène pratique, stratégie (voir à ce sujet Fontanille, 2008a). Pourtant, le travail sur les stratégies énonciatives ne s'est jamais constitué en une étude systématique de rhétorique: comme le remarque aussi le Groupe $\mu$ dans le cas de la 
sémiotique visuelle d'inspiration greimassienne, il s'agit le plus souvent d'analyses d'un système visuel ad hoc ${ }^{11}$, système qui n'est pas généralisable. D'ailleurs, la force de la sémiotique énonciative réside justement dans sa procédure caractérisante (dans le sens de Rastier), qui permet de retracer finement les parcours de sens thésaurisés et supportés par les configurations textualisées ${ }^{12}$. Si elle possède des instruments très efficaces pour l'analyse suprasegmentale de textes visuels, ces mêmes instruments ne permettent pas de concevoir un modèle rhétorique d'inspiration grammaticale, constitué par des unités disjointes, dont la composition serait réglementée et généralisable ${ }^{13}$.

Pour revenir à la question de la stylisation et du geste, il faut remarquer qu'une rhétorique du geste pictural (mais aussi sculptural et peut-être photographique) est encore à venir. D'ailleurs, à ce sujet, le Groupe $\mu$ a donné des suggestions importantes: pensons aux différents types de transformation qui ont mis en valeur les stratégies de filtrage, de discrétisation, etc., et qui permettent par exemple d'esquisser une typologie des textures possibles du trait (en tant que résultat d'un acte de traçage), en faisant de ce dernier une zone de différentiation d'épaisseur plastique et non une simple différentiation ponctuelle (ligne). La prise en compte de la genèse du trait permet, par conséquent, de rendre pertinents les rythmes de la main et des instruments du peintre et les résistances des matériaux du support inscrit. Par ce biais, une rhétorique du geste pourrait être envisageable, en dépassant le niveau atomique des unités et prenant comme taille d'analyse les éléments suprasegmentaux, tels les rythmes, leurs régularités, leurs aspectualités d'appréhension.

Si la rhétorique des figures est bien une rhétorique des unités ${ }^{14}$ - l'invariant étant le maintien d'une partie commune entre les deux degrés, conçu et perçu -, d'ailleurs difficiles à identifier dans un certain nombre de tableaux abstraits par exemple, et si l'autre rhétorique concerne les stratégies d'assomption, elles pourraient converger toutes deux vers une rhétorique des prépositions (voir Bordron, ici même), des pronoms et des adverbes visuels, ce qui leur permettrait de constituer non seulement une rhétorique du geste énonciatif (avec des fonctions grammaticales), mais aussi une rhétorique des tons. En fait, ce que les deux rhétoriques du visuel ont laissé en suspens est le fait que les images peuvent assumer un ton plaisant, solennel, sarcastique, etc. Comment pouvoir expliquer que certaines images fonctionnant rhétoriquement de la même manière, selon les opérations énumérées par le Groupe $\mu$, ne partagent pourtant pas les mêmes tons? Le travail sur les tons devrait forcément partir d'une étude sur l'énonciation, et sur les relations entre assertion et assomption: l'image a la capacité de se manifester à travers le plan énonciatif de l'assomption, elle peut assumer de manière contrastive ce qu'elle affirme sur le plan dénotatif, elle peut se déclarer elle-même comme véritable, erronée, fausse, infidèle; elle se manifeste enfin comme une confession épistémique à côté de ce qu'elle asserte/représente. Mais on ne peut pas se contenter de cela. Comme l'affirme Pierluigi Basso Fossali, qui a travaillé sur les figures tropiques et les tons dans les films de David Lynch,

[...] la sémantique des tons profile en somme une rhétorique au carré, ou de second ordre, où les différentes isotopies sur le plan de l'énonciation non seulement peuvent coexister et être concurrentielles, mais il arrive aussi qu'elles échangent à l'improviste de rôle hiérarchique (une allotopie peut devenir dominante sur les isotopies de fond).

(2006: 210; nous traduisons)

Certes, on ne peut pas réduire la sémantique des tons à la question de l'intensité des assomptions concurrentielles des valeurs énoncées par des instances de l'énonciation ${ }^{15}$. Comme l'affirme encore Basso Fossali, une taxonomie des tons peut trouver différents ancrages discursifs: il y a des tons qui relèvent de la teneur du discours (colloquiale, institutionnelle, etc.), de la manière d'appeler l'énonciataire en cause (ton menaçant, provocateur), de la manière de s'exprimer (ton exalté, humble, etc.), de la manière de décrire le monde ambiant et l'expérience (ton ampoulé, sec, etc.), de profiter du canal pour activer le spectateur (ton sonore, etc.), d'arranger la prosodie (ton traînant, etc.). L'analyse du ton permettrait de raffiner et de stratifier la complexité énonciative, car elle a comme enjeu de rendre compte de la congruence plus ou moins réussie de toutes ses facettes ${ }^{16}$. La sémantique 
des tons couple la dramatisation de la distribution des valeurs avec celle des valences, à savoir la manière dont les valeurs, non seulement de l'énoncé mais aussi de l'énonciation elle-même, sont rhétoriquement aménagées.

\section{LA RHÉTORIQUE DES TROUS NOIRS ENTRE SCIENCE ET ART}

Si on reprend la définition que donne aujourd'hui Jean-Marie Klinkenberg de la rhétorique en tant que «négociation de la distance sémiotique entre partenaires» (2008: 39), on pourrait affirmer que, dans le discours scientifique, c'est la vulgarisation savante qui est le genre discursif le plus intéressant à étudier de ce point de vue. La vulgarisation est le genre rhétorique par excellence, car elle doit savoir construire une plasticité cognitive, voire une ergonomie cognitive des objets de la recherche pour en faire des objets déformables, manipulables et transposables dans des représentations le plus possible concevables dans le monde de la vie quotidienne. Les ouvrages de vulgarisation savante sont les lieux des arrangements cognitifs par excellence: ils sont lus par des collègues de la même discipline qui ne sont pas des spécialistes de la matière spécifique traitée. Ces ouvrages doivent cependant pouvoir être lus aussi par des collègues d'autres disciplines limitrophes. On voudrait aborder cette manipulabilité des objets scientifiques selon les différents genres discursifs, en prenant en considération deux publications portant sur les trous noirs, qui nous offrent un exemple très intéressant d'ergonomie cognitive et d'arrangement rhétorique.

Comme il est couramment admis, il est impossible de filmer ou de photographier les trous noirs: ils sont décrits comme une sorte de gouffre qui attire la lumière (un rayon lumineux, par exemple, y est complètement absorbé et tout ce qui approche un trou noir y disparait). Les trous noirs sont donc non seulement théorisés comme des manifestations invisibles, mais leur existence n'est que supposée à partir d'autres phénomènes auxquels il faut trouver une source ou une explication. On les appelle justement "a theoretical object", soit un objet théorique dont la configuration dépend de la théorie de la relativité générale et d'autres hypothèses que les équations rendent opérationnelles.

Jean-Pierre Luminet a produit et publié en 1979 une photographie calculée des trous noirs en en proposant ainsi une première iconographie (image 1).

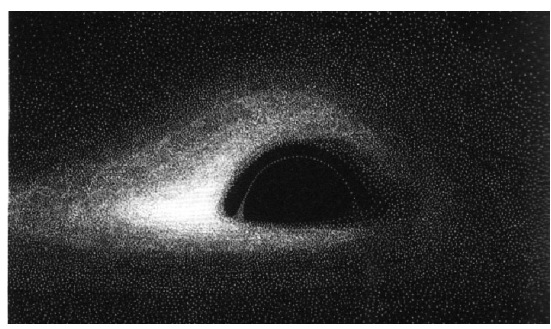

Image 1

"Apparence lointaine d'un trou noir sphérique entouré d'un disque d'accrétion ". Photographie virtuelle d'un trou noir, calculée en 1978 sur ordinateur (reprise dans Luminet, 2006 : 284). Image reproduite avec l'autorisation de l'auteur.

Cette iconographie a été calculée à partir d'équations (image 2) comme on le voit dans l'article de recherche ayant pour titre «Image of a Spherical Black Hole with Thin Accretion Disk».
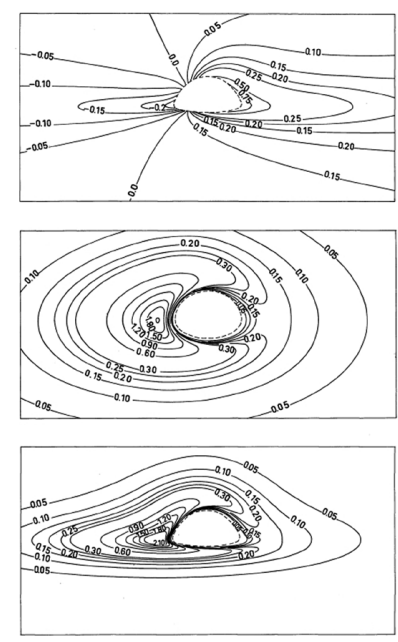

Image 2

"Courbes du disque d'accrétion selon différents points d'observation" (Luminet, 1979 : 234). Image reproduite avec l'autorisation de l'auteur.

Dans l'article en question, l'iconographie finale (image 1) des trous noirs (au pluriel) est justifiée et se construit pas à pas à travers des calculs mathématiques ${ }^{17}$. Au contraire, dans les ouvrages de vulgarisation, les images des trous noirs sont censées représenter la vision fictive qu'un observateur 
tombant dans un trou noir pourrait avoir. En fait, dans les trois chapitres de l'ouvrage de vulgarisation de Jean-Pierre Luminet, Le Destin de l'univers. Trous noirs et énergie sombre (2006), on est face à un tout autre parcours d'argumentation et d'explication de l'iconographie et du fonctionnement des trous noirs que dans les articles de recherche. La différence la plus macroscopique entre les deux textes concerne évidemment le fait que les équations ont totalement disparu de cet ouvrage: l'ensemble des images et schémas rencontrés dans ces trois chapitres sont des visualisations qui ont perdu toute référence stricte à des procédés mathématiques d'instanciation et ne sont pas des images reproductibles à travers des paramètres expérimentaux connus. Il devient d'autant plus intéressant d'étudier comment et pourquoi des scientifiques comme Luminet utilisent des images artistiques qui se présentent comme des insertions allotopiques au sein de l'isotopie scientifique. Comme on le sait, les images artistiques ne sont jamais censées donner des explications sur leur acte productif, et elles sont des images uniques et originales, voire non reproductibles: tout cela va à l'encontre de la fabrication et de l'utilisation scientifique des images ainsi que de leur "crédibilité». Pourquoi donc les utiliser pour renforcer l'argumentation scientifique ${ }^{18}$ ? Comment une image ou une série d'images qui proviennent d'une tradition culturelle et d'un domaine social comme l'art, et qui se présentent comme statutairement allotopiques ${ }^{19}$ dans le cadre d'un discours scientifique, peuvent jouer un rôle dans la communication de la science, mais aussi dans la formulation de la pensée scientifique elle-même ${ }^{20}$ ? Comment l'image artistique peut-elle concourir au développement de la créativité scientifique?

Les images artistiques insérées dans l'argumentation scientifique sur lesquelles on voudrait attirer l'attention sont au nombre de deux. La première s'inspire du système de représentation de l'artiste hollandais Maurits Escher et est retravaillée par Luminet (image 3) qui, de l'artiste, reprend l'espace construit sur des non-congruences topologiques effrayantes et sur des architectures illogiques et non praticables pour bien montrer que le trou noir est un espace dévorant et duquel rien ne pourrait ressortir.

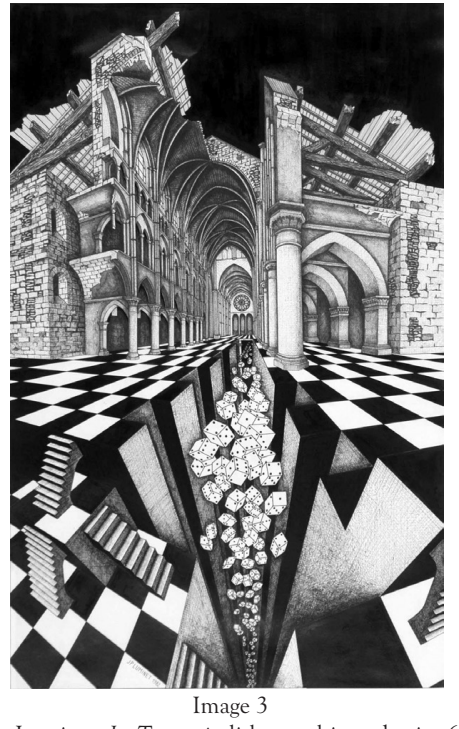

Jean-Pierre Luminet, Le Trou noir, lithographie et dessin, 66 x 91cm, 1992 Image reproduite avec l'autorisation de l'auteur.

D'ailleurs, Escher est l'artiste qui, plus que tout autre, a su décrire l'ouverture vers le vide, la montée qui se transforme en une chute aspirée; en un mot, il est l'artiste du vertige, ce qui convient bien à la mise en scène des trous noirs.

L'autre image, un dessin de A. Rackham extraite d'un conte de l'écrivain E.A. Poe contenu dans Tales of Mystery and Imagination (image 4), représente un vortex.

Dans l'image d'après Escher (image 3), nous sommes placés dans la position de spectateurs voyant un gouffre s'ouvrir à leurs pieds. Dans l'image de

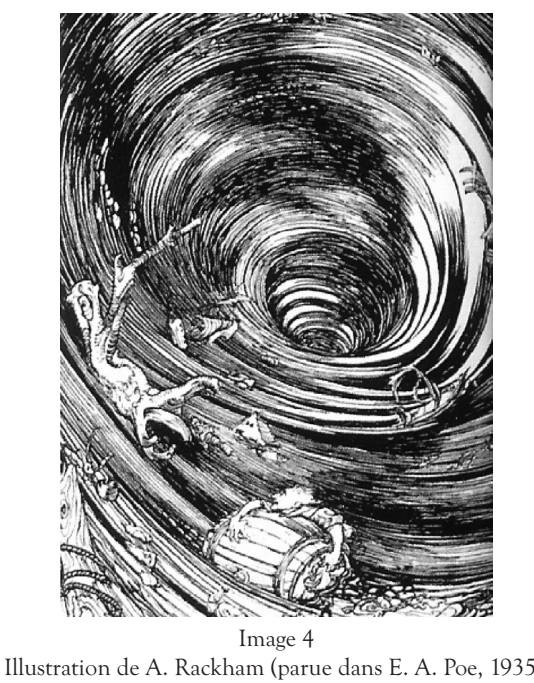


Rackham (image 4), nous sommes déjà plongés dans un stade ultérieur de la chute: la perspective est inclusive; nous, les spectateurs, sommes déjà entraînés par le remous et perdons le sens du bas et du haut. Ici le tourbillon est irrésistible.

Nous voyons que, progressant d'une image artistique à l'autre, ainsi qu'en progressant d'une citation littéraire à l'autre ${ }^{21}$, la description de la chute se précise de plus en plus, ainsi que les effets sur notre perception. Les deux images racontent la même histoire - il s'agit dans les deux cas d'une histoire visuelle extrême exprimant la peur de l'abyme sans fond -, mais elles la racontent à travers deux formes aspectuelles différentes (la première inchoative, la deuxième ponctuelle) et des tempos différents (la première sur le mode du parvenir, l'autre sur le mode du survenir).

C'est l'image d'après Escher qui paraît la plus intéressante d'un point de vue rhétorique, et c'est pour cette raison que nous voudrions nous y arrêter brièvement.

Les figures des dés mettent en scène la tentative scientifique de brider le hasard en le rapportant à des résultats contrôlables (les six faces du cube). Ils représentent des instruments de calcul de ce qui se passe et qui n'est pas entièrement maîtrisable. Emportés par la force attractive du trou noir, les dés tombent dans une fissure tectonique et ils montrent ainsi le point-limite de la calculabilité scientifique: à l'intérieur de ce gouffre, il manquera un plan d'appui et ils ne pourront plus, probablement, se stabiliser en une position qui manifeste un résultat. Ces dés sont donc une métonymie de tous les éléments prothétiques de l'être humain qui visent à brider l'indétermination. Le trou noir est au contraire un mouvement chaotique qui ne permet même pas une position d'observation statistique de ses turbulences. Aucune maîtrise de cet espace n'est possible: toute position des dés ou de l'observateur est provisoire, fuyante. Les faces des dés deviennent de plus en plus indiscernables plus on descend dans le puits. Leur pluralisation met encore plus en valeur leur fonctionnement en tant que matériaux pour construire et édifier des architectures théoriques possibles: leur chute est la débâcle de toute construction.

\subsection{L'exemplification figurale}

On pourrait affirmer, en suivant la doxa, qu'à l'origine de toute hypothèse scientifique, dans le cas des objets théoriques, il y a le monde mathématique des équations, et qu'ensuite il y a la vulgarisation, faite de citations littéraires ${ }^{22}$ et d'images artistiques. Mais on s'aperçoit, en lisant l'ouvrage de Luminet, que ces dernières ne visent pas à simplifier les prises de position théorique: elles visent au contraire à exemplifier des conflits de force grâce à une transposition figurative et plastique qui permet de rendre perceptibles ce que les théories physico-mathématiques rendent pertinentes dans le cas des trous noirs: les tensions entre perte d'orientation et recherche de repères, entre engloutissement et stabilisation proprioceptive. Les images rendent analogiquement saisissables des perceptions possibles. Possibles au sens où nous ne les avons jamais éprouvées dans leur manifestation ultime - et d'ailleurs les trous noirs ne sont que des hypothèses d'objets possibles -, mais qui font pourtant part de nos sentiments les plus intimes. Il s'agit de modèles culturels qui, tout en ne décidant pas de l'iconographie scientifique (elle a été calculée comme on le voit dans les images 1 et 2), permettent d'envisager d'abord la concevabilité de la théorie qui produit les hypothèses à vérifier et seulement ensuite la cascade d'équations qui, de leur côté, produiront l'iconographie calculée.

Les iconographies, celles obtenues par calcul et celles du discours artistique, ont une histoire génétique différente, mais elles se rejoignent lorsque l'iconographie de base des trous noirs (image 1), calculée à travers des équations, se transforme en un dispositif formé de plusieurs images fonctionnant comme la visualisation d'une simulation de voyage dans un trou noir (image 5).

Ces vortex, dont on contrôle les paramètres et qui peuvent donc se définir comme des images de laboratoire, s'apparentent de près aux iconographies des tourbillons offertes par les images artistiques. Cela advient lorsque le scientifique a besoin, à partir des images calculées, de simuler quel pourrait être l'effet de l'engloutissement progressif pour le corps humain, qui reste toujours l'échelle fondamentale de toute recherche scientifique. 

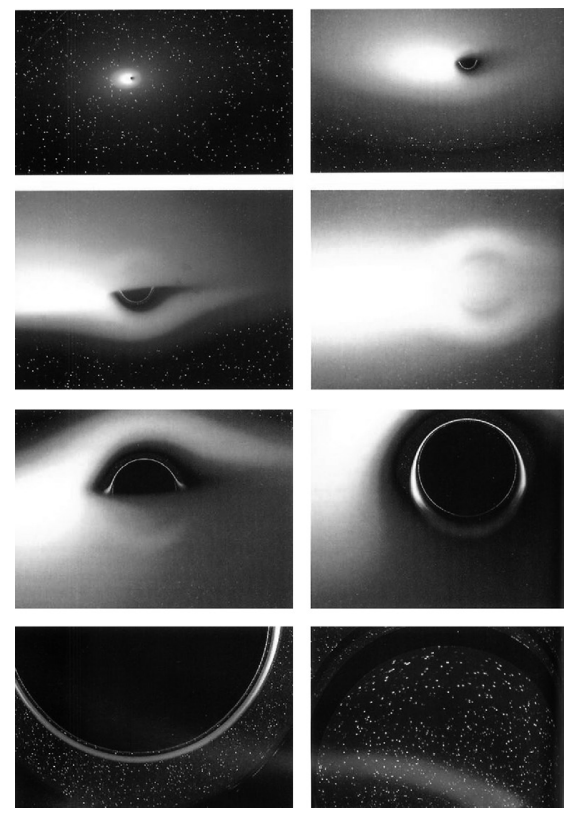

Image 5

"Le plongeon d'un astronaute intrépide dans un trou noir " (Luminet, 2006 : 288). Image reproduite avec l'autorisation de l'auteur.

D'une certaine manière, on pourrait dire que ces dernières images proposent une iconographie qui résout l'écart statutaire entre le domaine de l'art et le domaine de la science. Il nous semble en fait que l'iconographie artistique vient en aide au parcours strictement scientifique du premier article de Luminet en 1979, parce qu'elle permet de contribuer à l'exemplification figurale du fonctionnement des trous noirs. La notion d'exemplification figurale se fonde sur l'exemplification métaphorique de Nelson Goodman (1992) qui a essayé de substituer la centralité de la notion de dénotation, en tant que renvoi du langage à un monde de référence, par le concept d'exemplification. Comme l'affirme Basso Fossali (2008), le concept d'exemplification nous permet d'envisager le médium linguistique (au sens large, sémiotique) comme un passage essentiel pour constituer des échantillons d'un monde auquel on ne peut attribuer une signification qu'en le constituant. C'est le cas de nos images artistiques, qui permettent de concevoir/constituer un monde auquel la connaissance expérimentale n'a pas d'accès. Les images artistiques doivent donc être conçues comme des objets culturels fonctionnant comme des échantillons de monde qui exemplifient des propriétés d'un objet (la vitesse de rotation, l'effet de tourbillon, la non-possibilité de retour, etc.). Bien sûr, l'image artistique ne possède pas les mêmes possibilités de généralisation et de génération des équations qui permettent, elles, de calculer la vitesse de rotation, etc., ni le partage du même domaine de valeurs; de plus, ces équations rendent opaque la projection des propriétés exemplifiées dans l'ensemble théorique qu'elles devraient contribuer à constituer. Mais cette opacité se révèle comme justifiable à partir d'un point de vue rhétorique: la nature hétérogène de l'exemplification figurale est constitutive et sa vocation est celle de se projeter vers un scénario "autre", en gardant en mémoire son potentiel sémantique. D'ailleurs, tout scénario de valeurs, associé à un domaine social (scientifique, artistique, religieux, etc.), ne se suffit pas à lui-même et l'exemplification figurale est censée remplir les manques de bases et de fondements de chaque domaine. L'opacité et la charge allotopique des iconographies artistiques nous demandent d'activer une tension entre scénarios différents: c'est comme si un point de vue anamorphique permettait de déplacer la noncongruence du paysage figuratif des images artistiques dans un autre paysage (celui de l'image calculée) qui l'englobe et l'intègre pour rejoindre une nouvelle intelligibilité, un espace tiers qui n'est pas intratextuel, mais bien intertextuel.

L'image artistique offre à la science des trous noirs une "expériencialité» virtuelle: elle devient le support d'une expérience, d'une réversion expérimentale. Si normalement, pour détecter des phénomènes épars qui pourraient concourir à l'identification d'un phénomène comparable aux hypothèses du trou noir, on utilise les prothèses et les instruments les plus éloignés et abstraits du corps humain (l'astronomie gamma en l'occurrence), l'image artistique peut assurer, à travers une anaphore fulgurante, un retour à l'incarnation. C'est pour cela que l'image artistique, en étant "perspectivée», devient domestique et ré-embrayable à travers les coordonnées déictiques qu'elle offre. L'image artistique incarne la perceptionaction de parvenir enfin à se rapprocher du trou noir qui se charge ainsi de valeur relationnelle: sa valeur devient celle de pouvoir le rejoindre. 


\section{POUR CONCLURE:}

l'origine artistique de la réflexion scientifique

Dans l'article de recherche que Luminet avait rédigé en 1979, l'attention portée sur la relation entre équations et visualisations se concentrait sur la recherche des liens et des justifications entre les étapes du processus de référentialisation. Dans ce cas, la référence en question était construite par la théorie de la relativité générale, partagée par tous les collègues qui étaient censés lire l'article, et il ne fallait pas la justifier en tant que source de l'article sur les trous noirs. Dans l'ouvrage de 2006, au contraire, la communauté des possibles lecteurs s'élargit, et c'est pour cela que Luminet tend à construire une véritable archéologie de l'être concevable même du trou noir: toutes les références à la littérature et à l'histoire de la culture se justifient à travers cette nécessité de remonter à l'origine de la référentialisation, une origine partageable. On s'aperçoit ainsi que, dans la vulgarisation, il s'agit non plus de rendre explicites les connexions et les liens entre les étapes du parcours de référentialisation, mais bien au contraire de justifier en amont l'origine d'une hypothèse et d'en trouver les traces dans notre univers culturel.

Paradoxalement, l'image artistique permet au lecteur de l'ouvrage scientifique de faire un pas en arrière sur les fondements de ces recherches: si, dans l'article de recherche, les fondements de la référentialisation ne concernent que la théorie de la relativité générale et les équations qui en découlent, dans l'ouvrage de vulgarisation, les fondements de la référentialisation vont plus loin et concernent des configurations sémiotiques fondées sur des formes de vie fondamentales de la culture occidentale. Ces images artistiques, allotopiques par rapport à la procédure strictement scientifique, mettent en évidence le fait que Luminet réfléchit sur l'origine culturelle de l'image fabriquée à la fin des années 1970 et sur les savoirs stratifiés dans la compétence du lecteur en tant que source de sa théorisation.

\section{NOTES}

1. Selon l'acception de Fontanille (1999), l'assertion est l'acte d'énonciation par lequel le contenu d'un énoncé est identifié comme étant dans le champ de présence du discours, l'assomption au contraire est autoréférentielle: à travers le niveau de l'assomption, l'image fait connaître sa position par rapport à ce qui advient dans son champ. Outre l'assertion (graduation des modes d'existence des valeurs) et l'assomption (intensité d'embrayage), Fontanille envisage encore une troisième composante, la déclinaison figurative qui concerne l'extension en termes d'élasticité discursive. Bien sûr la figure, en tant qu'allotopique, concerne la relation entre un paysage énoncé et le mouvement énonciatif qui le rend non homogène, mais la rhétorique visuelle du Groupe $\mu$ n'a jamais pensé cette relation en termes de modulation d'embrayage plus ou moins fort des valeurs en opposition. 2. Une exception importante est Denis Bertrand. En ligne: http:// denisbertrand.unblog.fr/textes-en-ligne/semiotique-et-rhetorique (page consultée le 3 février 2010).

3. Comme l'affirme Fontanille dans un article récent, cette approche «vise à intégrer la rhétorique dans une théorie des discours, comme une composante spécifique, certes, mais soumise aux mêmes règles que les autres composantes discursives »; et ensuite: "[c]es catégories [discursives, sur lesquelles opèrent les figures et les tropes] pourraient, à peu de frais semble-t-il, être généralisées, et considérées comme les catégories de la praxis énonciative elle-même " (2008b: 18). Et encore: "au lieu de traiter la rhétorique comme un répertoire, un système ou une macro-structure de règles et de normes de la production des tropes et figures, on la considérera comme une des dimensions du processus énonciatif" (ibid.: 19).

4. Sur la figure comme argumentation, voir Klinkenberg (2000, 2008). 5. À ce propos, Jean-François Bordron a écrit dans un article consacré à W. James et au pragmatisme qu'on pourrait trouver les mêmes principes rhétoriques de compositions entre les parties et le tout ou selon les parties entre elles (dissociations, mélanges, inclusions, etc.) dans le discours et dans la composition interne du flux perceptif. Sur cette notion de rhétorique de l'expérience, voir son article «Le pragmatisme et l'innovation esthétique. Réflexions sur la nature du changement" (à paraître) et sur la rhétorique comme gestuelle, voir Bordron (ici même). Sur la construction des mondes et de la connaissance en général, voir Goodman (1992): le philosophe explique comment la construction des mondes (dans les domaines de l'art, de la science, de la perception, du langage ordinaire) passe par des actions telles que la composition et la décomposition, la pondération (accentuation), l'agencement, la suppression et la supplémentation, la déformation, etc.

6. Des études importantes ont été faites dans ce sens, mais surtout à partir de la rhétorique verbale (Klinkenberg, 1990).

7. Fontanille propose lui aussi une manière de lier les deux approches en question, celle des figures et celle de la praxis énonciative (les catégories discursives telles que l'intensité, la quantité, le conflit et l'assomption n'étant pas des catégories spécifiques du fonctionnement rhétorique, mais de la praxis énonciative en général): «on doit pouvoir retrouver, dans la définition même des figures de rhétorique, des catégories discursives comme le "conflit", l'"intensité" et l'"étendue". Il nous faut en somme retrouver, dans le "répertoire" et le "système" rhétorique des figures et des tropes, le modèle catégoriel et syntagmatique de leurs effets en discours" (2008b: 20).

8. Pour l'auteure, le scientifique est un opérateur textuel et intertextuel qui utilise des critères de composition et de décomposition de la réalité physique et discursive. Elle analyse comment le style scientifique est construit afin de prévoir des critiques, de répondre, en avance, implicitement aux objections possibles, etc. À ce propos, Bastide remarque que tout texte scientifique construit des controverses internes 
implicites: tout texte est un contre-texte, toute position, une opposition, toute preuve, une contre-preuve, toute affirmation théorique, la négation d'une autre théorie précédente. Le texte scientifique condense en soi des programmes et des anti-programmes (qui peuvent être des matériaux, d'autres durées d'expérimentations, etc.) qui réfléchissent une situation conflictuelle externe au discours (controverses scientifiques).

9. D'autant moins que la photographie, malheureusement, a souvent été étudiée comme une image instantanée par rapport aux effets de sens (générativité), alors qu'elle est instantanée seulement par rapport à la production (genèse). Pour une réflexion à cet égard, voir Floch (1986) et Dondero (2009a).

10. Voir Groupe $\mu$ (1992), Beyaert-Geslin $(2004 ;$ 2008), Le Guern (2004; 2008). De plus, sur le rapport entre énonciation énoncée, énonciation en acte et praxis énonciative, voir Beyaert-Geslin, Dondero et Fontanille (2009).

11. Le Groupe $\mu$ a essayé de dépasser cette difficulté en théorisant, dans le cadre de la rhétorique visuelle, un degré zéro local et un degré zéro général; ce dernier lui permet d'intégrer une typologie de configurations de traits plastiques existants au-delà des énoncés, dans lesquels ces configurations se manifestent, et de formuler un système grammatical justement externe aux énoncés, ce que la sémiotique greimassienne n'a jamais voulu faire.

12. L'objectif de l'analyse greimassienne est en fait d'atteindre une description des parcours sémantiques (et perceptifs) activés par les textes singuliers, autrement dit de construire une validité des descriptions négociable au niveau intersubjectif pour objectiver " une trajectoire, définie a minima, des processus de sémantisation activables par le récepteur " (Basso Fossali, 2003: 24-25; nous traduisons et soulignons) et rendre opératoire "un cadre descriptif négociable comme arrièrefond intersubjectif tendu à la commensurabilité de nos diverses interprétations" (ibid.).

13. Si, dans la sémiotique greimassienne, ce qui est ad hoc est le système des traits à analyser, qui ne s'appuie pas sur un système grammatical externe à l'énoncé (ce qui, pour la sémiotique du Groupe $\mu$, correspond au type plastique), la sémiotique du Groupe $\mu$, par contre, sélectionne de manière ad hoc les exemples sur lesquels elle généralise le fonctionnement, par exemple, de la rhétorique de la forme (voir l'exemple heureux de Vasarely). Si ces exemples ne développent pas un pouvoir herméneutique aussi fort que ceux de la rhétorique de l'énonciation - ils servent notamment à expliquer des fonctionnements généraux -, la logique de cette architecture de cas de figure a le mérite de construire une cartographie des fonctionnements non seulement existants, mais aussi possibles.

14. «Parler de partie commune suppose que l'élément figuré puisse être décomposé en unités d'ordre inférieur. C'est cette possibilité d'articulation qui permet d'identifier l'invariant, grâce à une évaluation des compatibilités entre la base et l'élément figuré " (Groupe $\mu$, 1992 : 264).

15. D'après Basso Fossali, le ton peut «allier un caractère global avec un local: en effet, il peut être entendu à la fois comme une détermination du global sur le local (le ton détermine des choix paradigmatiques et des connexions syntagmatiques, par exemple en termes de lexique et de prosodie) et comme une inflexion locale qui, de manière presque sibylline, permet de ré-interpréter le faire énonciatif (et avec lui sa compétence modale et ses programmes narratifs)»(2006: 210; nous traduisons).

16. À ce propos, Basso Fossali (2006) distingue le ton du style, qui lui aussi relève de la présence de l'énonciateur par rapport à son discours. Le style concerne de près une classe d'invariants transtextuels et intrapoétiques, les tons en sont justement des variables, des endroits textuels où le style "fait des caprices".
17. Pour une explication plus détaillée du fonctionnement des équations par rapport à la visualisation, voir Dondero (2010, à paraître).

18. Il faut pourtant préciser qu'il s'agit de types de démonstration et d'utilisation d'images qui changent en concomitance avec les genres discursifs pris en considération: normalement, on ne trouve pas d'images artistiques dans les articles de recherche; chez les astrophysiciens, on en trouve dans des ouvrages à ambition généraliste ou dans les ouvrages de vulgarisation savante.

19. L'allotopie est la distance sémantique prise par rapport à une valeur attendue. Si l'isotopie concerne la présence de mêmes valeurs catégorielles, à l'intérieur d'un texte, qui en fondent la cohérence, l'allotopie concerne la présence concessive de valeurs figuratives qui apparaissent comme non congruentes par rapport au fond sur lequel elles surviennent. Dans ce cas-ci, il ne s'agit pas d'une allotopie discursive, mais d'une allotopie statutaire: l'image artistique, dont la procédure de production est autographique et sacralisée, est utilisée dans le cadre du discours scientifique qui prescrit, quant à lui, à l'image un fort contrôle des paramètres de fabrication et une complète reproductibilité par d'autres scientifiques.

20. Voir à ce sujet l'ouvrage majeur de Pierantoni (2003) et le prochain numéro de la revue Semiotica, «L'image entre art et science: une sémiotique de l'attirance», dirigé par A. Beyaert-Geslin et M. G. Dondero.

21. Toute la théorisation de Luminet dans cet ouvrage est accompagnée, et même, on pourrait dire, se fonde sur des citations provenant des plus célèbres poètes et romanciers de tous les temps. Dante: «Vous qui entrez ici, laissez toute espérance" (Luminet, 2006: 260); E.A. Poe: "Je fus possédé de la plus ardente curiosité relativement au tourbillon lui-même. Je sentis positivement le désir d'explorer ses profondeurs, même au prix du sacrifice que j'allais faire; mon principal chagrin était de penser que je ne pourrais jamais raconter à mes camarades les mystères que j'allais connaître" (ibid.: 264); «Mais il me reste peu de temps pour rêver à ma destinée! Les cercles se rétrécissent rapidement - nous plongeons follement dans l'étreinte du tourbillon - et, à travers le mugissement, le beuglement et le détonement de l'Océan et de la tempête, le navire tremble - oh! Dieu! - il se dérobe, il sombre!» (ibid.: 268); Rimbaud: «La plaque du foyer noir, de réels soleils de grèves: ah! puits de magies" (ibid.: 277).

22. Le texte d'explication d'un fonctionnement d'un trou noir est accompagné par des légendes, des exergues, des encadrés, comme dans le cas du poème d'E. A. Poe. Ce qui est plus étonnant, c'est que tout au long de la description du voyage fictif près du centre du tourbillon du trou noir, à savoir tout au long du texte principal de l'explication, Luminet cite des extraits des contes de E. A. Poe (1935). En fait, Luminet attaque la description en affirmant: «Alors, à $20 \mathrm{~km} / \mathrm{h}$, les cercles des navigations plongent tellement dans la gueule du tourbillon que, comme l'écrit E. A. Poe "si un navire entre dans la région de son attraction, il est inévitablement absorbé et entrainé au fond et, là, déchiré en morceaux" " $(2006: 270)$. On voit bien que la fiction littéraire peut offrir ses mots au récit scientifique.

\section{RÉFÉRENCES BIBLIOGRAPHIQUES}

Allamel-RAFFIN, C. [2010]: «Un exemple d'étude comparée des procédures interprétatives à l'œuvre dans les sciences de la nature et dans l'analyse des œuvres d'art », à paraître dans Semiotica.

BAsso Fossali, P. [2003]: Confini del cinema. Strategie estetiche e ricerca semiotica, Turin, Lindau;

[2006] : Interpretazione tra mondi. Il pensiero figurale di David Lynch, Pise, ETS;

[2008] : La promozione dei valori. Semiotica della comunicazione e dei consumi, Milan, Franco Angeli. 
BASTIDE, F. [1985]: «Iconographie des textes scientifiques: principes d'analyse", Culture technique, ${ }^{\circ} 14,132-151$;

_ [2001]: Una notte con Saturno. Scritti semiotici sul discorso scientifico, Rome, Meltemi.

BERTRAND, D. [2003]: «L'extraction du sens: instances énonciatives et figuration de l'indicible», Versants, no ${ }^{44-45}$, numéro spécial ("L'interprétation littéraire aujourd'hui»), 317-329;

[2007]: «L'expression rhétorique des matières", dans

D. Bertrand, M. Costantini, S. Dambrine et J. Alonso (dir.), La Transversalité du sens, Saint-Denis, PUV;

[2008]: «Entre catachrèse et métaphore: la figuration du discours ", dans U. Bähler (dir.), Sémiotique de la métaphore, Neuchâtel, La Baconnière;

[2009]: «La provocation figurative de la métamorphose», dans

M. Colas-Blaise et A. Beyaert-Geslin (dir.), Le Sens de la métamorphose, Limoges, Pulim.

BEYAERT-GESLIN, A. [2004] : «Texture, couleur, lumière et autres arrangements de la perception ", Protée, vol. 31 n³, 81-90;

_ [2008] : «De la texture à la matière», Protée, vol. 36 n², 101-110;

- [2009]: L'Image préoccupée, Paris, Hermès Lavoisier.

Beyaert-Geslin, A., M. G. Dondero et J. FonTANille (dir.) [2009]:

"Arts du faire: production et expertise", Nouveaux Actes Sémiotiques.

En ligne: http://revues.unilim.fr/nas/sommaire.php?id=3050 (page consultée le 3 février 2010).

DAMISCH, H. [2000]: «La peinture prise au mot», préface dans M. Schapiro, 5-28.

Dondero, M. G. [2009a]: Le Sacré dans l'image photographique. Études sémiotiques, Paris, Hermès Lavoisier;

[2009b]: «La sémiotique visuelle entre principes généraux et spécificités. À partir du Groupe $\mu$ ", Nouveaux Actes Sémiotiques. En ligne: http://revues.unilim.fr/nas/document.php?id=3286\#bodyftn 1 (page consultée le 3 février 2010);

[2010]: «L'autorialité de l'image scientifique et l'image artistique comme prophétie scientifique", à paraître dans Semiotica.

FABBRI, P. [2004]: «Étude de l'aquarelle En forme de sphinx de Paul

Klee», dans A. Hénault et A. Beyaert (dir.), Ateliers de sémiotique visuelle, Paris, PUF, 189-210;

[2009]: Le Tournant sémiotique, préface d'Y.Jeanneret, Paris, Hermès-Lavoisier.

FLOCH, J.M. [1986]: Les Formes des l'empreinte, Paris, Fanlac. FLORENSKIJ, P. A. [1995]: Lo spazio e il tempo nell'arte, Milan, Adelphi. FONTANILLE, J. [1996] : «Le trope visuel entre présence et absence ", Protée, vol. 24, n 1, 47-54;

[1999]: Sémiotique du discours, Limoges, Pulim;

__ [2008a]: Pratiques sémiotiques, Limoges, Pulim;

[2008b]: «La dimension rhétorique du discours: les valeurs

en jeu ", dans S. Badir et J.-M. Klinkenberg (dir.), Figures de la figure, Limoges, Pulim, 17-34.
GIGANTE, E. [2009]: Autoportraits en marge. Images de l'auteur dans la peinture de la Renaissance, thèse de doctorat, Paris, École des hautes études en sciences sociales.

GoOdman, N. [1992] : Manières de faire des mondes, Paris, Jacqueline Chambon.

GROUPE $\mu$ [1992]: Traité du signe visuel. Pour une rhétorique de l'image,

Paris, Seuil.

JACKSON, B. [1985]: Semiotics and Legal Theory, London, Routledge \&

Kegan Paul.

KLINKENBERG, J.-M. [1990]: «Rhétorique de l'argumentation et

rhétorique des figures", dans M. Meyer et A. Lempereur (dir.), Figures et

conflits rhétoriques, Bruxelles, Éd. de l'Université de Bruxelles, 115-137;

[2000]: «L'argumentation dans la figure", Cahiers de

praxematique, n०35, 59-86;

[2008] : «La rhétorique dans le sémiotique: la composante

créative du système", dans S. Badir et J.-M. Klinkenberg (dir.), Figures de la figure, Limoges, Pulim, 35-56.

LATOUR, B. et P. FABBRI [1977]: «La rhétorique de la science: pouvoir et devoir dans un article de science exacte", Actes de la recherche en sciences sociales, vol. 13, $\mathrm{n}^{\circ} 1,81-95$.

LE GUERN, O. [2004]: «Entre tactile et visuel: textiles et textures photographiques et picturales", dans A. Hénault et A. Beyaert (dir.), Ateliers de sémiotique visuelle, Paris, PUF, 171-188;

[2008]: De la parole à l'œuvre. Synthèse, thèse d'habilitation à

diriger des recherches, Université Lumière-Lyon 2.

LHOTE, A. [(1958) 1999]: Traités du paysage et de la figure, Paris, Grasset. LUMINET, J.-P. [1979]: «Image of a spherical black hole with thin accretion disk", Astronomy and Astrophysics, vol. 75, no 1-2, 228-235;

_ [2006]: Le Destin de l'univers. Trous noirs et énergie sombre, Paris, Fayard.

MARIN, L. [1993]: De la représentation, Paris, Gallimard.

PARIS, J. [1969]: L'Espace et le Regard, Paris, Seuil.

PIERANTONI, R. [2003]: Vortici, atomi e sirene. Immagini e forme del pensiero esatto, Milan, Mondadori Electa.

POE, E. A. [1935]: Tales of Mystery and Imagination, Londres, George G.

Harrap \& Co.

SCHAPIRO, M. [2000]: «Face et profil comme formes symboliques ", Les Mots et les Images, Paris, Macula, 93-124.

SHAÏRI, H.-R. et J. FONTANILLE [2001]: «Approche sémiotique du regard photographique: deux empreintes de l'Iran contemporain ", Nouveaux Actes Sémiotiques, n 73-75, 87-120.

SONESSON, G. [2007]: «La rhétorique de la perception. Recherche de méthode", dans S. Badir et J.-M. Klinkenberg (dir.), Figures de la figure, Limoges, Pulim, 103-132;

SONESSON, G. et M. G. DONDERO [2010] : «Le Groupe $\mu$. Quarante ans de rhétorique - Trente-trois ans de sémiotique visuelle», Nouveaux Actes Sémiotiques. En ligne: http://revues.unilim.fr/nas/document. php?id=3248 (page consultée le 3 février 2010). 